\title{
Prediction of Multiple Serum Tumor Markers in Hepatolithiasis Complicated with Intrahepatic Cholangiocarcinoma
}

\author{
Hua Zhao', Benliang Lu \\ 'Department of Hepatobiliary Surgery, Zhoukou Central Hospital, Zhoukou, Henan, People's Republic of China; ${ }^{2}$ Department of Clinical Laboratory, \\ Zhoukou Central Hospital, Zhoukou, Henan, People's Republic of China \\ Correspondence: Hua Zhao, Department of Hepatobiliary Surgery, Zhoukou Central Hospital, 26th East Renmin Road, Zhoukou, Henan, 466000, \\ People's Republic of China, Tel/Fax +86 3948208295, Email jhonazao@gmail.com
}

\begin{abstract}
Objective: To evaluate the predictive value of multiple serum tumor markers in hepatolithiasis complicated with intrahepatic cholangiocarcinoma (hepatolithiasis-associated cholangiocarcinoma, HL-CCA).

Methods: From January 2010 to December 2020, three hundred eighty-five hepatolithiasis patients in Zhoukou Central Hospital were retrospectively analyzed, among them thirty patients complicated with intrahepatic cholangiocarcinoma. The levels of serum AFP, CA125, CA19-9, CA242, and CEA in hepatolithiasis or HL-CCA were measured. Receiver operating characteristic (ROC) curves were used to evaluate the diagnostic effects of single or combined detection of the five tumor markers for HL-CCA.

Results: The level of AFP was insignificantly different between the hepatolithiasis and HL-CCA $(P=0.730)$. CA125, CA19-9, CA242 and CEA were elevated in HL-CCA. The area under ROC curves (AUCs) of the single detection of serum CA125, CA19-9, CA242 and CEA were all more than 0.5. The maximum AUC occurred in CA19-9. The AUC of AFP was slightly higher than 0.5, but the level of serum AFP was insignificantly different between hepatolithiasis and HL-CCA. The single detection of AFP was not of great significance to the differential diagnosis. The optimal cut-off values of CA125, CA19-9, CA242, and CEA were 104.09 kU/L, 383.28 U/mL, 152.56 kU/L, 6.29 $\mathrm{ng} / \mathrm{mL}$. The diagnostic effect of CA19-9 was the highest one for differential diagnosis between hepatolithiasis and HL-CCA. With reference to CA19-9 $>383.28 \mathrm{U} / \mathrm{mL}$, the sensitivity and specificity of the single detection were $80.00 \%$ and $82.61 \%$ respectively, and the AUC was 0.883. The diagnostic effect of CEA was secondary toward CA19-9. With reference to CEA $>6.29 \mathrm{ng} / \mathrm{mL}$, the sensitivity and specificity of the single detection were $66.67 \%$ and $83.76 \%$ respectively, and the AUC was 0.814 .
\end{abstract}

Conclusion: Serum CA19-9 maybe the effective tumor marker in the diagnosis of HL-CCA, and measurement of combined serum tumor markers can help in the detection of HL-CCA.

Keywords: hepatolithiasis, cholangiocarcinoma, tumor marker, ROC curve

\section{Introduction}

Hepatolithiasis is a rare disease in the majority of western world countries, and proportion of hepatolithiasis to all cholelithiasis cases vary from $1 \%$ to $2.2 \%$. But hepatolithiasis is prevalent in East Asia, including China mainland, with incidence rates ranging from $2 \%$ to $25 \%$ to all cholelithiasis cases. ${ }^{1,2}$

Cholangiocarcinoma has increased globally over the past few decades. ${ }^{3}$ Hepatolithiasis is an established risk factor for intrahepatic cholangiocarcinoma. ${ }^{4}$ Other established risk factors are parasitic infections, primary sclerosing cholangitis (PSC), biliary-duct cysts, and toxin. The clinical progression of hepatolithiasis may lead to liver parenchymal destruction due to recurrent cholangitis. Furthermore, hepatolithiasis can subsequently result in biliary cirrhosis. Although the process of carcinogenesis from hepatolithiasis is not fully understood, it has been proposed to be a multi-step process, involving hyperplasia, dysplasia, and adenocarcinoma in situ to invasive adenocarcinoma. Among them, chronic proliferative cholangitis plays a role in biliary carcinogenesis. ${ }^{5}$ Recurrent cholangitis, biliary stricture, bile stasis, and chronic bacterial infection are common phenomena in 
hepatolithiasis. These recurrent or chronic inflammatory events cause prolonged inflammation of the bile duct epithelium and can lead to the development of cholangiocarcinoma.

The clinical manifestations HL-CCA are nonspecific, though they may be abdominal pain, fever, fatigue, night sweats, etc. ${ }^{6}$ It is difficult to detect early intrahepatic cholangiocarcinoma that occurs as a complication of hepatolithiasis. A careful search for the presence of cholangiocarcinoma is crucial in the treatment of patients with hepatolithiasis. To date, preoperative imaging workup, especially with CT, is important for the selection of appropriate surgical therapy and treatment planning in HL-CCA. However, there are many limitations in differentiate intrahepatic cholangiocarcinoma from fibrosis in hepatolithiasis. It is difficult to differentiate stricture, infiltrating type of intrahepatic cholangiocarcinoma, mass-forming cholangiocarcinoma, and inflammatory pseudo-tumor because prolonged affected liver segments often become fibrotic and scarred. Intrahepatic cholangiocarcinoma is hard to distinguish from inflammatory mass complicated with hepatolithiasis in clinical practice preoperatively. ${ }^{5}$

Tumor markers are useful tools for screening and diagnose cancers in high-risk patients. Furthermore, tumor markers can also evaluate the response to cancer treatment and detect the recurrence of cancer. Elevated concentration of serum CA19-9 in intrahepatic cholangiocarcinoma has been frequently reported. ${ }^{7-10}$ CA19-9 levels can distinguish between benign and malignant pancreaticobiliary diseases. ${ }^{11,12}$ Multiple studies have suggested that elevated serum concentration of CA19-9 was significantly related the prognosis in patients with intrahepatic cholangiocarcinoma, ${ }^{13-15}$ while others also demonstrated that both CA19-9 and CEA levels could be independent prognostic factors. ${ }^{16}$ But few studies focused on the changes of serum tumor markers in HL-CCA, and the purpose of the present study was to observe the changes of multiple serum tumor markers in HL-CCA. The results are as follows.

\section{Materials and Methods General Data}

From January 2010 to December 2020, three hundred eighty-five hepatolithiasis patients in Zhoukou Central Hospital were retrospectively analyzed, among them thirty patients complicated with intrahepatic cholangiocarcinoma. All patients were confirmed by laboratory examination, imaging examination and final clinicopathological diagnosis. Exclusion criteria in this study: (1) patients with special medical records within one month, such as liver failure, kidney failure, etc.; (2) recently take drugs that can affect liver function; (3) patients with the diagnosis of other cancers or immune diseases, such as PSC; (4) patients with acute cholangitis or jaundice. There were two groups. One group was only hepatolithiasis, and another group was HL-CCA.

We compared the two groups with regard to clinical features such as: gender, age, Alanine Aminotransferase (ALT), Aspartate Transaminase (AST), and ALkaline Phosphatase (ALP). And tumor markers, Alpha-fetoprotein (AFP), carcinoembryonic antigen (CEA), carbohydrate antigen 125 (CA125), carbohydrate antigen 19-9 (CA19-9), carbohydrate antigen 242 (CA242) were also measured.

The fasting venous blood $(5 \mathrm{~mL})$ of patients in the morning was drawn and centrifuged at $3000 \mathrm{r} / \mathrm{m}$ for 10 minutes. The serum was packaged into the sterile test tube at $-80^{\circ} \mathrm{C}$ fridge for preservation. Serum levels of AFP, CA125, CA19-9, CA242, and CEA were analyzed within 4 hours after collection. Electrical chemiluminescent immunoassay (ECLIA) was used by a Roche automatic analyzer and associated kits (Roche, Switzerland). This study was conducted in accordance with the ethical standards of the World Medical Association Declaration of Helsinki and was approved by the Ethics Committee of the Zhoukou Central Hospital. Due to the retrospective nature of the study and lack of interference with treatment, the requirement for written informed consent for participation was waived. In addition, our research data are confidential.

\section{Statistical Analysis}

SPSS 28.0 (IBM SPSS, Inc, Chicago, IL, USA) and MedCalc 20.011 (MedCalc Software, Ltd, Ostend, Belgium) software were used to analyze data and realize visualization. Two independent rank sample sum test (Mann-Whitney $U$-test) was used to analyze and expressed as a median and inter-quartile range (IQR). Chi-square or Fisher's exact test was used as appropriate to analyze the categorical data expressed as frequency (percentage). The cutoff values were calculated by ROC curves. Combined detection of the tumor markers was evaluated in a logistic regression model. $P<$ 0.05 was considered statistically significant. 
Table I Comparison of the Hepatolithiasis $(\mathrm{HL})$ and $\mathrm{HL}-\mathrm{CCA}(\overline{\mathrm{X}} \pm \mathrm{s}, \%)$.

\begin{tabular}{|l|l|l|l|l|l|}
\hline & Age (Years) & Gender (Male/Female) & Serum ALT (U/L) & Serum AST (U/L) & Serum ALP (U/L) \\
\hline HL $(n=355)$ & $62.6 \pm 4.2$ & $121 / 234$ & $121 \pm 27.2$ & $89 \pm 24.2$ & $325 \pm 45.6$ \\
HL-CCA $(n=30)$ & $60.3 \pm 3.6$ & $12 / 18$ & $78 \pm 32.8$ & $82 \pm 23.6$ & $375 \pm 22.6$ \\
$P$ & 0.103 & 0.551 & 0.069 & 0.153 & 0.342 \\
\hline
\end{tabular}

Table 2 Comparison of Serum Tumor Markers Between the HL and HL-CCA (Median and IQR).

\begin{tabular}{|c|c|c|c|c|c|c|c|c|}
\hline & \multicolumn{3}{|c|}{ HL } & \multicolumn{3}{|c|}{ HL-CCA } & \multirow[t]{2}{*}{$\mathbf{z}$} & \multirow[t]{2}{*}{$P$} \\
\hline & Median & Percentile 25 & Percentile 75 & Median & Percentile 25 & Percentile 75 & & \\
\hline AFP(ug/L) & 3.920 & 2.000 & 11.520 & 4.660 & 2.255 & 11.523 & -0.345 & 0.730 \\
\hline CAI25(kU/L) & 33.775 & 19.190 & 102.748 & 89.380 & 24.943 & 281.875 & -1.984 & 0.047 \\
\hline CAI9-9(U/mL) & 157.000 & 30.250 & 333.595 & 641.120 & 386.500 & 829.360 & -4.933 & $<0.001$ \\
\hline CA242(kU/L) & 41.210 & 10.000 & 114.540 & 175.820 & 89.090 & 281.040 & -3.608 & $<0.001$ \\
\hline $\mathrm{CEA}(\mathrm{ng} / \mathrm{mL})$ & 3.500 & $\mathrm{I} .725$ & 4.945 & 11.060 & 4.505 & 32.793 & -5.017 & $<0.001$ \\
\hline
\end{tabular}

\section{Results}

\section{Characteristics of Patients}

The main characteristics of the two groups were insignificant (Table 1). The main age and gender ratio were similar between the two groups. There was no significant difference in liver function test between the two groups, such as ALT, AST, ALP. The morbidity of HL-CCA among all hepatolithiasis was $0.078(30 / 385)$.

\section{Comparison of Serum Tumor Markers Between the Two Groups}

The five variables did not conform to normal distribution, and the tendency of dispersion was presented as the median and IQR. The level of serum AFP was insignificantly different between the two groups $(P=0.730)$. CA125, CA19-9, CA242 and CEA were elevated in HL-CCA (Table 2).

\section{Evaluation of Diagnostic Effect of Single Detection of the Five Serum Tumor Markers} AUCs of the single detection of serum CA125, CA19-9, CA242 and CEA were all more than 0.5 (Table 3, Figure 1B-E). The single detection of five tumor markers was described in the AUCs as follows (Figure 1F). The maximum AUC occurred in CA19-9 (Figure 1C). The AUC of AFP was slightly higher than 0.5 (Figure 1A), but the level of AFP was insignificantly different between the two groups. The single detection of AFP was not of great significance to the differential diagnosis. According to ROC curves, the optimal cut-off values (associated criterion) of CA125, CA19-9, CA242, and CEA were $104.09 \mathrm{kU} / \mathrm{L}, 383.28 \mathrm{U} / \mathrm{mL}, 152.56 \mathrm{kU} / \mathrm{L}, 6.29 \mathrm{ng} / \mathrm{mL}$. The diagnostic effect of CA19-9 was highest for differential diagnosis between the two groups. With reference to CA19-9 $>383.28 \mathrm{U} / \mathrm{mL}$, the sensitivity and specificity of the single detection were $80.00 \%$ and $82.61 \%$, respectively, and the AUC was 0.883 (Figure 1C). The diagnostic effect of CEA was secondary toward CA19-9. With reference to CEA $>6.29 \mathrm{ng} / \mathrm{mL}$, the sensitivity and specificity of the single detection were $66.67 \%$ and $83.76 \%$, respectively, and the AUC was 0.814 (Figure 1E).

Table 3 Evaluation of Single Detection of AFP, CA125, CA19-9, CA242 and CEA in HL-CCA (\%).

\begin{tabular}{|l|l|l|l|l|}
\hline & Cut-off Value & Sensitivity & Specificity & AUC \\
\hline AFP & $>3.5 \mathrm{ug} / \mathrm{L}$ & 66.67 & 44.93 & 0.512 \\
CA125 & $>104.09 \mathrm{kU} / \mathrm{L}$ & 57.69 & 74.62 & 0.670 \\
CA19-9 & $>383.28 \mathrm{U} / \mathrm{mL}$ & 80.00 & 82.61 & 0.883 \\
CA242 & $>152.56 \mathrm{kU} / \mathrm{L}$ & 68.42 & 85.38 & 0.753 \\
CEA & $>6.29 \mathrm{ng} / \mathrm{mL}$ & 66.67 & 83.76 & 0.814 \\
\hline
\end{tabular}



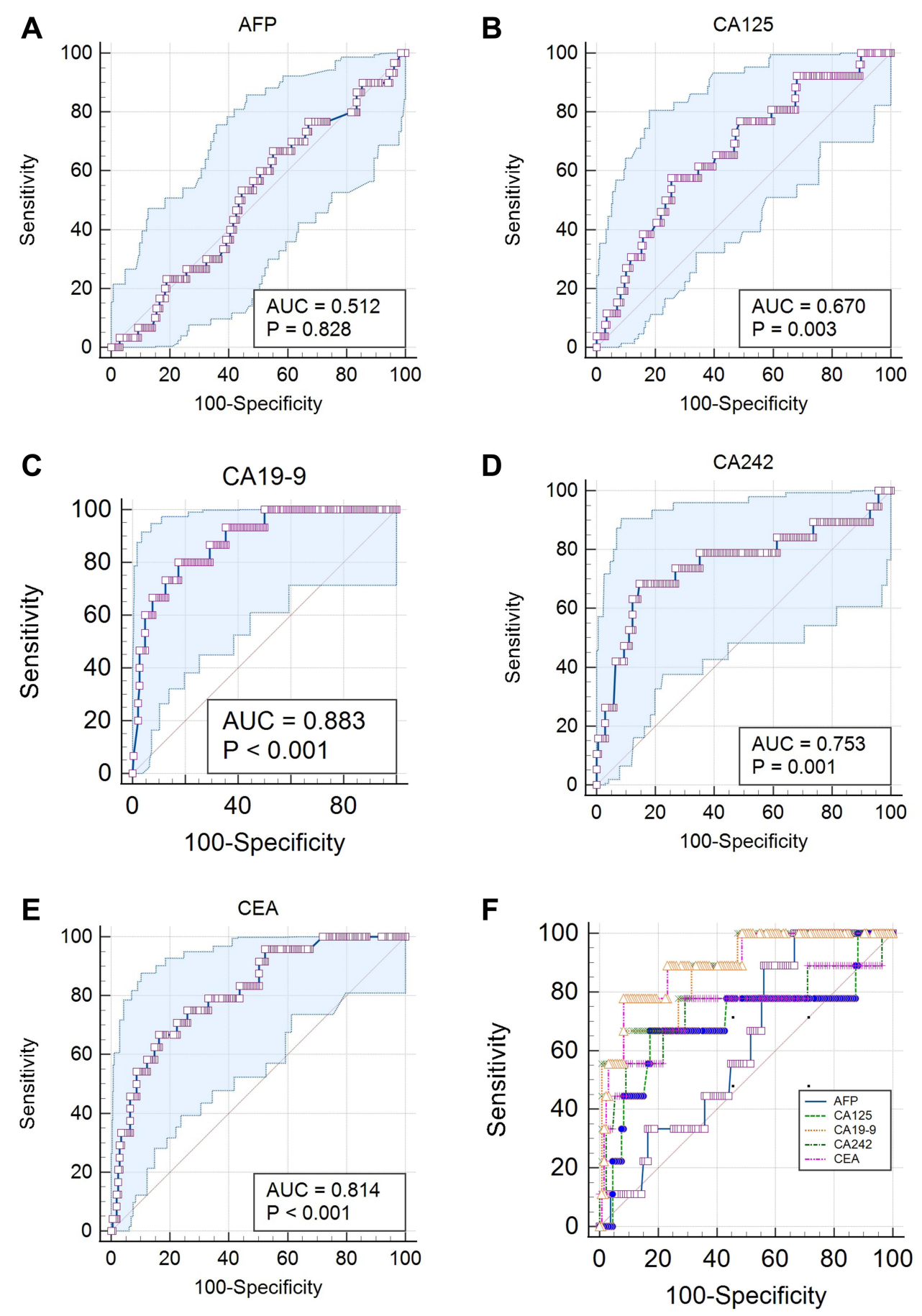

Figure I (A) ROC curve for AFP in HL-CCA. (B) ROC curve for CAI25 in HL-CCA. (C) ROC curve for CAI9-9 in HL-CCA. (D) ROC curve for CA242 in HL-CCA. (E) ROC curve for CEA in HL-CCA. (F) ROC curves for combined tumor markers in HL-CCA.

\section{Evaluation of Diagnostic Effect of Combined Detection of the Five Serum Tumor Markers}

The highest AUC occurred in the combined detection of CA19-9, CA125 and CA242 (0.910, 95\% CI: 0.853 to 0.950). However, the combination of these three markers had only a small improvement toward with the combination of CA19-9 and CA125 (0.901, 95\% CI: 0.848 to 0.940$)$ (Table 4). 
Table 4 Evaluation of Combined Detection of AFP, CA125, CA19-9, CA242 and CEA in the Diagnosis HL- CCA (\%).

\begin{tabular}{|l|l|l|}
\hline & AUC & $\mathbf{9 5 \%} \mathbf{C l}$ \\
\hline CA125+CA19-9 & 0.901 & 0.848 to 0.940 \\
CA125+CA242 & 0.777 & 0.709 to 0.835 \\
CA125+CEA & 0.759 & 0.695 to 0.815 \\
CA19-9+CA242 & 0.893 & 0.836 to 0.936 \\
CA19-9+CEA & 0.882 & 0.826 to 0.925 \\
CA242+CEA & 0.811 & 0.746 to 0.866 \\
CA19-9+CA125+CA242 & 0.910 & 0.853 to 0.950 \\
CA19-9+CA242+CEA & 0.881 & 0.819 to 0.928 \\
CA125+CA242+CEA & 0.798 & 0.730 to 0.856 \\
CA19-9+CA125+CEA & 0.899 & 0.844 to 0.940 \\
CA125+CA19-9+CA242+CEA & 0.895 & 0.834 to 0.940 \\
\hline
\end{tabular}

\section{Discussion}

Because HL-CCA lacks specific clinical manifestations and is easy to be covered up by the clinical manifestations of cholelithiasis, most of the patients are in the middle or late stage when they firstly visited the hospital. ${ }^{17}$ This makes the early diagnosis of the disease more difficult.

Macroscopically, intrahepatic cholangiocarcinomas have been classified further as being either (1) a mass-forming type, (2) a periductal infiltrating type, or (3) an intraductal growth type, with the most commonly encountered types being the desmoplastic infiltrating nodular or diffusely infiltrating varieties. ${ }^{18}$ The detection of HL-CCA is dependent on imaging modalities mainly, such as ultrasonography (US), CT, and MRI. Preoperative imaging workup, especially with $\mathrm{CT}$, is important for the selection of appropriate surgical therapy and treatment planning. Typical multi-phase CT findings of cholangiocarcinoma are parenchymal low-attenuated mass with rim enhancement, capsular retraction and delayed enhancement, biliary duct wall thickening, or intraductal polypoid mass with dilatation of upstream bile ducts. ${ }^{19}$ However, from the imaging findings, fibrotic masses in the bile duct walls and periductal hepatic parenchyma are often seen as a form of periductal tumor-like lesions and may appear similar to cholangiocarcinoma. Furthermore, in patients with hepatolithiasis, the stricture caused by stones may not be easy to differentiate from that caused by cholangiocarcinoma. Unfortunately, unresectable disease (13.3\%) is found at the time of exploration despite extensive preoperative evaluation, thus resulting in unnecessary laparotomy. ${ }^{6}$

Tumor markers are substances present in or produced by cancer cells or other cells of the body in response to cancer or certain benign (noncancerous) conditions that provide information about a cancer. Because some of these substances can be detected in body samples such as blood, urine, and tissue, these markers may be used to help detect and diagnose some types of cancer, predict and monitor a person's response to certain treatments, and detect recurrence. ${ }^{20}$ In this study, specific tumor markers were elevated in HL-CCA patients, and the combined detection of multiple tumor markers was helpful to predict tumor occurrence.

In recent decades, AFP is still the most widely used tumor marker for screening hepatocellular carcinoma (HCC). Dysregulated levels of AFP in the plasma strongly correlate with HCC malignancy. ${ }^{21-23}$ Serum AFP assessments for HCC surveillance, in combination with US are recommended as diagnostic tools according to the Asian HCC guidelines. ${ }^{24}$ However, AFP has little value in the diagnosis of intrahepatic cholangiocarcinoma. ${ }^{25}$ Our experiment also confirmed that the level of serum AFP was insignificantly different between hepatolithiasis and HL-CCA.

CA125 also known as mucin 16 or MUC16 is a protein that in humans is encoded by the MUC16 gene. CA125 has found application as a tumor marker that may be elevated in the blood of some patients with specific types of cancers, or other conditions that are benign. In addition to ovarian cancer, CA-125 can be elevated in patients who have conditions such as endometrial cancer, fallopian tube cancer, lung cancer, breast cancer, and gastrointestinal cancer. ${ }^{26}$ In our study, the AUC of CA125 was 0.670 and the optimal cut-off was $104.09 \mathrm{kU} / \mathrm{L}$. The sensitivity and specificity were $57.69 \%$ and 
$74.62 \%$, respectively. Except for AFP, the sensitivity and specificity of CA125 among the four tumor markers were the lowest.

CA19-9 is the most commonly used and best validated serum tumor marker for pancreatic cancer in symptomatic patients and for monitoring therapeutic improvement. ${ }^{27-29}$ Among the biliary tract diseases, CA19-9 can also be effective tumor marker of diagnosis of cholangiocarcinoma and monitoring the efficacy. ${ }^{30,31}$ Moreover, the application of CA19-9 can differentiate cancer from benign biliary diseases. ${ }^{12}$ In our present study, the sensitivity and specificity were $80.00 \%$ and $82.61 \%$, respectively. And AUC was 0.883 . It should be emphasized that biliary obstruction in benign conditions, more CA19-9 can also be secreted and leaked out into blood stream. ${ }^{11}$ Moreover, CA19-9 elevated in other diseases without evidence of malignant or pancreatobiliary diseases. ${ }^{32}$ The estimated causative diseases were hepatic diseases, pulmonary diseases, gynecologic diseases, endocrine diseases, and spleen diseases. As CA19-9 is a non-specific serum tumor marker for digestive system neoplasms, it is necessary to set the exact cut-off value. In our experiment, the cut-off value of CA19-9 was 383.28 U/L. Compared with other scholars' reports, ${ }^{25}$ it is relatively higher.

CA242 is a sialic acid-containing carbohydrate antigen attached to core proteins/lipids detected on the cell surface or in serum. CA242 have been used clinically as diagnostic biomarker for pancreatic, colorectal, and other cancers. ${ }^{33}$ It is commonly tested along with CEA and CA19-9 for detecting pancreatic cancer. Tao LY ${ }^{34}$ found that combined detection of AFP and CA242 can improve the specificity and accuracy of diagnosing intrahepatic cholangiocarcinoma. Among the four tumor markers, the sensitivity and specificity of CA242 were all in the middle.

CEA are glycosyl phosphatidyl inositol (GPI) cell-surface-anchored glycoproteins whose specialized sialofucosylated glycoforms serve as functional colon carcinoma L-selectin and E-selectin ligands, which may be critical to the metastatic dissemination of colon carcinoma cells. CEA is also one of the earliest confirmed tumor markers associated with intrahepatic cholangiocarcinoma. ${ }^{4}$ Surprisingly, Loosen $\mathrm{SH}^{35}$ found that CEA showed a higher accuracy for the differentiation between cholangiocarcinoma and patients with PSC compared to CA19-9. Furthermore, only CEA but not CA19-9 represented an independent predictor of survival in multivariate Cox-regression analysis.

To compensate for the defect ability of prediction, combination of multiple tumor markers is advocated to improve the sensibility and specificity of hepatobiliary tumors. ${ }^{36,37}$ In our study, the highest AUC occurred in the combined detection of CA19-9, CA125 and CA242.

\section{Conclusion}

In conclusion, our preliminary results suggest that serum CA19-9 is effective tumor marker in the diagnosis of HL-CCA, and measurement of combined serum tumor markers can help in the early detection of HL-CCA.

\section{Disclosure}

The authors declare no conflicts of interest in this work.

\section{References}

1. Tazuma S. Gallstone disease: epidemiology, pathogenesis, and classification of biliary stones (common bile duct and intrahepatic). Best Pract Res Clin Gastroenterol. 2006;20(6):1075-1083. doi:10.1016/j.bpg.2006.05.009

2. Shoda J, Tanaka N, Osuga T. Hepatolithiasis-epidemiology and pathogenesis update. Front Biosci. 2003;1(8):e398-e409. doi:10.2741/1091

3. Bergquist A, von Seth E. Epidemiology of cholangiocarcinoma. Best Pract Res Clin Gastroenterol. 2015;29(2):221-232. doi:10.1016/j. bpg.2015.02.003

4. Kubo S, Kinoshita H, Hirohashi K, et al. Hepatolithiasis associated with cholangiocarcinoma. World J Surg. 1995;19(4):637-641. doi:10.1007/ BF00294744

5. Kim HJ, Kim JS, Joo MK, et al. Hepatolithiasis and intrahepatic cholangiocarcinoma: a review. World J Gastroenterol. 2015;21(48):13418-13431. doi:10.3748/wjg.v21.i48.13418

6. Li HY, Zhou SJ, Li M, et al. Diagnosis and cure experience of hepatolithiasis-associated intrahepatic cholangiocarcinoma in 66 patients. Asian Pac J Cancer Prev. 2012;13(2):725-729. doi:10.7314/apjcp.2012.13.2.725

7. Silsirivanit A, Matsuda A, Kuno A, et al. Multi-serum glycobiomarkers improves the diagnosis and prognostic prediction of cholangiocarcinoma. Clin Chim Acta. 2020;510:142-149. doi:10.1016/j.cca.2020.07.01

8. Tshering G, Dorji PW, Chaijaroenkul W, et al. Biomarkers for the diagnosis of cholangiocarcinoma: a systematic review. Am J Trop Med Hyg. 2018;98(6):1788-1797. doi:10.4269/ajtmh.17-0879

9. Asaoka T, Kobayashi S, Hanaki T, et al. Clinical significance of preoperative CA19-9 and lymph node metastasis in intrahepatic cholangiocarcinoma. Surg Today. 2020;50(10):1176-1186. doi:10.1007/s00595-020-01992-x 
10. Liu Y, Sun J, Zhang Q, et al. Identification of bile survivin and carbohydrate antigen 199 in distinguishing cholangiocarcinoma from benign obstructive jaundice. Biomark Med. 2017;11(1):11-18. doi:10.2217/bmm-2016-0178

11. Kim MS, Jeon TJ, Park JY, et al. Clinical interpretation of elevated CA 19-9 levels in obstructive jaundice following benign and malignant pancreatobiliary disease. Korean J Gastroenterol. 2017;70(2):96-102. doi:10.4166/kjg.2017.70.2.96

12. Morris-Stiff G, Teli M, Jardine N, et al. CA19-9 antigen levels can distinguish between benign and malignant pancreaticobiliary disease. Hepatobiliary Pancreat Dis Int. 2009;8(6):620-626.

13. Kojima S, Hisaka T, Midorikawa R, et al. Prognostic impact of desmoplastic reaction evaluation for intrahepatic cholangiocarcinoma. Anticancer Res. 2020;40(8):4749-4754. doi:10.21873/anticanres.14476

14. Juntermanns B, Kaiser GM, Itani GS, et al. CA19-9 beim intrahepatischen Cholangiokarzinom: Ein diagnostisches und prognostisches Armamentarium? [CA19-9 in intrahepatic cholangiocarcinoma: a diagnostic and prognostic armamentarium?]. Chirurg. 2018;89(6):466-471. German. doi:10.1007/s00104-018-0636-z

15. Chen L, Zeng F, Yao L, et al. Nomogram based on inflammatory indices for differentiating intrahepatic cholangiocarcinoma from hepatocellular carcinoma. Cancer Med. 2020;9(4):1451-1461. doi:10.1002/cam4.2823

16. Qiang Z, Zhang W, Jin S, et al. Carcinoembryonic antigen, $\alpha$-fetoprotein, and Ki67 as biomarkers and prognostic factors in intrahepatic cholangiocarcinoma: a retrospective cohort study. Ann Hepatol. 2021;20:100242. doi:10.1016/j.aohep.2020.07.010

17. Lee JY, Kim JS, Moon JM, et al. Incidence of cholangiocarcinoma with or without previous resection of liver for hepatolithiasis. Gut Liver. 2013;7 (4):475-479. doi:10.5009/gnl.2013.7.4.475

18. Sirica AE. Cholangiocarcinoma: molecular targeting strategies for chemoprevention and therapy. Hepatology. 2005;41(1):5-15. doi:10.1002/ hep. 20537

19. Park HS, Lee JM, Kim SH, et al. CT differentiation of cholangiocarcinoma from periductal fibrosis in patients with hepatolithiasis. $A J R$ Am J Roentgenol. 2006;187(2):445-453. doi:10.2214/AJR.05.0247

20. Faria SC, Sagebiel T, Patnana M, et al. Tumor markers: myths and facts unfolded. Abdom Radiol. 2019;44(4):1575-1600. doi:10.1007/s00261-018$1845-0$

21. Luo P, Wu S, Yu Y, et al. Current status and perspective biomarkers in AFP negative HCC: towards screening for and diagnosing hepatocellular carcinoma at an earlier stage. Pathol Oncol Res. 2020;26(2):599-603. doi:10.1007/s12253-019-00585-5

22. Wang W, Wei C. Advances in the early diagnosis of hepatocellular carcinoma. Genes Dis. 2020;7(3):308-319. PMID: 32884985; PMCID: PMC7452544. doi:10.1016/j.gendis.2020.01.014

23. Ma WJ, Wang HY, Teng LS. Correlation analysis of preoperative serum alpha-fetoprotein (AFP) level and prognosis of hepatocellular carcinoma (HCC) after hepatectomy. World J Surg Oncol. 2013;11:212. doi:10.1186/1477-7819-11-212

24. Omata M, Cheng AL, Kokudo N, et al. Asia-Pacific clinical practice guidelines on the management of hepatocellular carcinoma: a 2017 update. Hepatol Int. 2017;11(4):317-370. doi:10.1007/s12072-017-9799-9

25. Li Y, Li DJ, Chen J, et al. Application of joint detection of AFP, CA19-9, CA125 and CEA in identification and diagnosis of cholangiocarcinoma. Asian Pac J Cancer Prev. 2015;16(8):3451-3455. doi:10.7314/apjcp.2015.16.8.3451

26. Scholler N, Urban N. CA125 in ovarian cancer. Biomark Med. 2007;1(4):513-523. doi:10.2217/17520363.1.4.513

27. Yako YY, Kruger D, Smith M, et al. Cytokines as biomarkers of pancreatic ductal adenocarcinoma: a systematic review. PLoS One. 2016;11(5): e0154016. doi:10.1371/journal.pone.0154016

28. Izumo W, Higuchi R, Furukawa T, et al. Evaluation of preoperative prognostic factors in patients with resectable pancreatic ductal adenocarcinoma. Scand J Gastroenterol. 2019;54(6):780-786. doi:10.1080/00365521.2019.1624816

29. Ge L, Pan B, Song F, et al. Comparing the diagnostic accuracy of five common tumour biomarkers and CA19-9 for pancreatic cancer: a protocol for a network meta-analysis of diagnostic test accuracy. BMJ Open. 2017;7(12):e018175. doi:10.1136/bmjopen-2017-018175

30. Lumachi F, Lo RG, Tozzoli R, et al. Measurement of serum carcinoembryonic antigen, carbohydrate antigen 19-9, cytokeratin-19 fragment and matrix metalloproteinase-7 for detecting cholangiocarcinoma: a preliminary case-control study. Anticancer Res. 2014;34(11):6663-6667.

31. Liang B, Zhong L, He Q, et al. Diagnostic accuracy of serum CA19-9 in patients with cholangiocarcinoma: a systematic review and meta-analysis. Med Sci Monit. 2015;21:3555-3563. doi:10.12659/msm.895040

32. Kim S, Park BK, Seo JH, et al. Carbohydrate antigen 19-9 elevation without evidence of malignant or pancreatobiliary diseases. Sci Rep. 2020;10 (1):8820. doi:10.1038/s41598-020-65720-8

33. Dou H, Sun G, Zhang L. CA242 as a biomarker for pancreatic cancer and other diseases. Prog Mol Biol Transl Sci. 2019;162:229-239. doi:10.1016/bs.pmbts.2018.12.007

34. Tao L-Y, Cai L, He X-D, et al. Comparison of serum tumor markers for intrahepatic cholangiocarcinoma and hepatocellular carcinoma. Am Surg. 2010;76(11):1210-1213. doi:10.1177/000313481007601119

35. Loosen SH, Roderburg C, Kauertz KL, et al. CEA but not CA19-9 is an independent prognostic factor in patients undergoing resection of cholangiocarcinoma. Sci Rep. 2017;7(1):16975. doi:10.1038/s41598-017-17175-7

36. Fang T, Wang H, Wang Y, et al. Clinical significance of preoperative serum CEA, CA125, and CA19-9 levels in predicting the resectability of cholangiocarcinoma. Dis Markers. 2019;2019:6016931. doi:10.1155/2019/6016931

37. Wongkham S, Silsirivanit A. State of serum markers for detection of cholangiocarcinoma. Asian Pac J Cancer Prev. 2012;13 Suppl:17-27.

Cancer Management and Research

Dovepress

Publish your work in this journal

Cancer Management and Research is an international, peer-reviewed open access journal focusing on cancer research and the optimal use of preventative and integrated treatment interventions to achieve improved outcomes, enhanced survival and quality of life for the cancer patient. The manuscript management system is completely online and includes a very quick and fair peer-review system, which is all easy to use. Visit http://www.dovepress.com/testimonials.php to read real quotes from published authors.

Submit your manuscript here: https://www.dovepress.com/cancer-management-and-research-journal 